\title{
Time horizon and choice by pigeons in a prey-selection task
}

\author{
C. M. S. PLOWRIGHT and SARA J. SHETTLEWORTH \\ University of Toronto, Toronto, Ontario, Canada
}

\begin{abstract}
In two operant simulations of prey selection, the choice behavior of pigeons and the predictions of a model of the effect of session length on optimal prey selection were compared. All but one of the predictions were confirmed. In the first experiment, a higher proportion of "bad" items (comparatively long delays to food) was accepted in 10-min sessions in one operant chamber than in the first $10 \mathrm{~min}$ of 20 -min sessions in a different chamber. The proportion of bad items accepted increased toward the end of the 20 -min sessions. In the second experiment, the birds were exposed both to 20-min sessions in one distinctively marked operant chamber and to sessions of variable length, with a mean of $20 \mathrm{~min}$, in another. A higher proportion of bad items was accepted in the variable-session chamber than in the fixed-session chamber at the beginning of the 20-min sessions. This difference decreased toward the end of the sessions but did not reverse as expected. In both experiments, an independent test analogous to the peak procedure was administered to establish whether the birds discriminated among the different session lengths.
\end{abstract}

The problem of how animals should select prey is one of the classic problems of optimal foraging theory (Stephens \& Krebs, 1986). In a prey-selection task (or diet-selection task), the forager searches for prey items, which it encounters one at a time. It must choose whether to accept or reject each encountered item. Items differ in their profitability (ratio of net energy yield to handling time $[e / h])$. When it is presented with prey items of two types, relatively good and bad (relatively high and low profitability, respectively), an energy-maximizing forager should always accept the relatively good items (relatively good and bad will heretofore be referred to simply as good and bad). The acceptance of the bad ones depends on whether the net rate of energy intake obtainable from such acceptance is higher than that obtainable from the animals' rejection of the bad items in favor of searching (see Stephens \& Krebs, 1986).

Several predictions of optimal prey selection regarding the effects of search time, profitability of good and bad prey items, and probability of encounter of good items have been tested in operant simulations (see Fantino \& Abarca, 1985; Shettleworth, 1988). In these operant simulations, the search time has been represented as a fixed-interval (Lea, 1979), a variable-interval (Abarca \& Fantino, 1982), or a variable-ratio (Hanson \& Green, 1989) schedule lead-

This research was supported by the Natural Sciences and Engineering Council of Canada through an operating grant to S.J.S. and a UCR grant from Saint Francis Xavier University to C.M.S.P. Some of the results in this paper were presented at the 30th annual meeting of the Psychonomic Society in Atlanta in 1989. We thank Jeff Lucas, Ken Cheng, and Peter Killeen for helpful comments on the manuscript, Erik Mathon and Orah Burack for help in collecting and analyzing the data, and Joanne Johnson and Chris Plowright for help in producing the figures. Correspondence may be addressed to C. M. S. Plowright, Ecole de Psychologie, Université d'Ottawa, Ottawa, Ontario K1N 6N5, Canada. ing to the presentation of a prey item. Good and bad items have been represented as large versus small food items (Krebs, Erichsen, Webber, \& Charnov, 1977; Lucas, 1987), as long versus short access time to food (Snyderman, 1983a), and as long versus short intervals or ratios leading to reinforcement (Hanson \& Green, 1989; Lea, 1979; Yoerg \& Kamil, 1988). In general, choice in these experiments has been close to optimal, in that the proportion of relatively bad items accepted increases as the density (probability of encounter) of relatively good items decreases. However, contrary to the predictions of the simple optimal-diet model, the acceptance of bad items generally does not increase in an all-or-nothing manner. At intermediate densities, partial preferences are observed. A number of possible reasons for these partial preferences have been suggested (McNamara \& Houston, 1987). For example, they could result from error or variability in the forager's estimate of prey density or quality. In the experiments reported here, we tested pigeons with a set of prey qualities and densities so that about $50 \%$ of the relatively bad items would be accepted, enabling us to observe possible changes in the acceptability of bad items (as described below) within foraging sessions.

Recent studies have stressed the importance of the time available for foraging, the "time horizon," as an important variable in diet selection (Lucas, 1985, 1987, 1990), as well as in other foraging tasks (Houston, Kacelnik, \& McNamara, 1982). The basic prediction for the effects of time horizon on prey selection follows from the fact that poor items should be rejected if the animal can expect to encounter and consume a better item during the time it would take to handle a bad item. However, if the forager is about to reach the end of a foraging bout, it can do no better than take the bad item at hand, since there will be no opportunity to encounter more good ones. 
Thus, when the time horizon is known to the forager, more bad items should be accepted toward the end of a session. Note that the term "time horizon" does not describe a property of the animal but is rather an operational definition referring to session length.

Several recent experiments have shown that foraging behavior is influenced by the amount of time available in a session. Swennen, Leopold, and deBruijn (1989) adjusted the tidal regime for oystercatchers and found an effect of time horizon on the time spent handling prey and the time spent searching. At least three studies have specifically reported an effect of time horizon on the acceptability of relatively bad items to foragers. Blue jays (Yoerg \& Kamil, 1988) that were given 10 - versus 20 -min sessions and great tits (Lucas, 1987) that were given 30versus 60-sec sessions were more likely to accept bad items toward the end of the short sessions than at the end of the same time period within the long sessions. Barnard and Hurst (1987) also found that foraging shrews were less selective (i.e., accepted a higher ratio of bad to good items) throughout 3- and 6-min sessions than they were over the same time period in 9-min sessions. These results confirm the predictions of the optimality analysis by Lucas (1985): By accepting a bad item at the end of a short session, the animal does not sacrifice an opportunity for a good item, as it does when it has more time available (see Stephens \& Krebs, 1986, for a discussion of the principle of lost opportunity).

In this paper, we report on two experiments in which we investigated the effects of session length on prey selection by pigeons. In the first experiment, we examined the effects of differences in session length (10 vs. $20 \mathrm{~min}$ ). In the second experiment, we examined the effects of variability of session length (20-min sessions vs. sessions of variable length, with a mean of $20 \mathrm{~min}$ ).

If animals adjust their choice as a function of session time, it should also be possible to show independently that they can discriminate among session lengths. In both experiments, an effort was made to relate the birds' performance on the choice task to their ability to discriminate among session lengths. Studies on the effects of time horizon on choice have not attempted to measure the animals' time perception independently of their choice behavior (Dow \& Lea, 1987; Shettleworth \& Plowright, 1989). The only exception of which we are aware is a study of the performance of rats foraging for food in temporally separated patches by Timberlake, Gawley, and Lucas (1987), who attempted to measure both foraging effort (suppression of food intake in the first patch) and anticipation of future food (early entry into the second patch). However, as noted by Timberlake et al., the two measures were not independent, since early entry into the second patch may have resulted in less foraging in the first.

Although there is now a large literature on temporal discrimination by animals in a number of situations (Church, 1978; Gibbon \& Allan, 1984), much of it deals with intervals of a few seconds or minutes (e.g., Drey- fus, Fetterman, Smith, \& Stubbs, 1988). Session length has been overlooked as a time interval to which animals may be sensitive. Nevertheless, ingenious procedures for investigating temporal discrimination can be adapted to study sensitivity to session lengths. In the "peak procedure" of Catania (1970) and Roberts (1981), rats were given two kinds of trials. Fixed interval (FI) trials were reinforced on an FI schedule, and empty trials lasted at least twice as long as the FI trials and no food was presented. On these empty trials, the rate of responding was found to increase and then decrease, with a peak response rate at the value of the FI, showing that the rats expected food at that time. When the standard FI was $40 \mathrm{sec}$ rather than $20 \mathrm{sec}$, the peak response rate occurred at $40 \mathrm{sec}$, but the shape of the response curve was less peaked: There were more responses between 20 and $60 \mathrm{sec}$ when the standard was $40 \mathrm{sec}$ than there were between 0 and $40 \mathrm{sec}$ when the standard was $20 \mathrm{sec}$. This result can be interpreted as an instance of Weber's law: The judgment of a 20-sec time interval is more accurate than that of a 40-sec time interval.

By analogy with the peak procedure, at the end of each session in our experiment, the search key remained on for a few minutes, but responses no longer led to the presentation of prey items. According to Weber's law, if the birds were sensitive to the session length in the first experiment, they should have responded at a lower rate or for less time after a 10-min session than after a 20min session. In the second experiment, in which session length was variable, the procedure was used to determine if the birds could discriminate among sessions of various lengths. If the birds expected an average duration of $20 \mathrm{~min}$, then they should have responded at a higher rate or for a longer duration at the end of sessions that were shorter than $20 \mathrm{~min}$, and at a lower rate or for a shorter duration at the end of sessions that were longer than $20 \mathrm{~min}$.

\section{EXPERIMENT 1}

The aim of this experiment was to determine whether pigeons could adjust their foraging behavior in a preyselection task in response to different session lengths. Short and long FI schedules represented, respectively, the good and bad items. Even though the food items delivered at the end of the short and long FIs were the same, the two schedules represented relatively good and bad items insofar as the ratios of net energy yield to time $(\mathrm{e} / \mathrm{h})$ differed. The experimental design incorporated some improvements over previous studies. In Yoerg and Kamil's (1988) study on blue jays, the delays to food for bad items varied from day to day, so that the probability of accepting a bad items was maintained at .5 in the middle of their sessions. Moreover, these delays (which they term "handling time') varied between short and long sessions. In Experiment 1, the delays were held constant. Also, because of possible long-term changes in choice behavior (Snyderman, 1983a), a long and a short session were ad- 
ministered each day, each in a different operant chamber, instead of having several days of long sessions followed by several days of short.

As described above, the search key remained on at the end of each session, and the rate of responding was taken as a measure of temporal discrimination. A further test of session-length discrimination was given at the end of the experiment. The short sessions were identical to the regular 10-min sessions. In the long sessions, however, the search key stopped yielding prey items after $10 \mathrm{~min}$ rather than the usual $20 \mathrm{~min}$. If the birds did indeed expect a long session, then they should have responded to the search key either at a higher rate or for a longer duration after $10 \mathrm{~min}$ in these test sessions than they did in the short sessions.

\section{Method}

Subjects. Subjects were 4 White King pigeons with various preexperimental histories. All had keypecked before. The birds were kept at $85 \% \pm 2 \%$ of their free-feeding body weight and were given mixed grain, as necessary, approximately $1 \mathrm{~h}$ after each experimental session. Water and grit were constantly available, except during the testing period. Two other birds began the experiment but were discarded during pretraining because of their low rates of responding.

Apparatus. Two $29.5 \times 30 \times 30 \mathrm{~cm}$ high operant chambers were used. On the side panel were three circular keys, $2 \mathrm{~cm}$ in diameter, placed in a row $6.5 \mathrm{~cm}$ from the top. The two side keys were each $4.5 \mathrm{~cm}$ from the center key. Eleven centimeters below each side key was a pellet dispenser connected to an $8 \times 7 \times 4 \mathrm{~cm}$ food tray, which could be illuminated by a white light. The key and the pellet dispenser on the right were nonoperational. The center key, which will be referred to as the search key, could be transilluminated by a white light. The left key, which will be referred to as the prey key, could be transilluminated with either red or green light by a projector.

In one box, the panel with the keys was covered with blue construction paper; in the other, it was covered with brown construction paper

An Apple Ile computer controlled the experimental contingencies and recorded the animals' behavior.

Pretraining and Procedure. Two of the birds were pretrained in each box. Pretraining consisted of four phases. The procedure during pretraining and during the experimental sessions is summarized in Table 1.

In Phase 1, the birds were autoshaped to peck the search key, even though all had keypecked before. The purpose of this phase was to have the pigeons keypeck reliably in the unfamiliar chambers.

In Phase 2, which lasted between six and eight sessions, they were given .5- to 1-h sessions in which they were exposed to both colors of light on the prey key but not the search key. A peck to either key was followed by an FI schedule, after which the prey-key light was extinguished, the feeder light was switched on for $4 \mathrm{sec}$, and four 20-mg Noyes pigeon pellets were delivered consecutively into the food tray. On the first day, a peck to the prey key, when it was red, was followed by four pellets after an FI schedule of $1 \mathrm{sec}$ (FI 1), and a peck to the prey key, when it was green, was followed by the same reinforcer after FI 6 . The FI requirement was subsequently increased to FI 2 for the red light and to FI 12, in increments of $2 \mathrm{sec}$, for the green light. A presentation of the red light on the prey key will be referred to as a presentation of a good item; a presentation of the green light on the prey key will be referred to as a presentation of a bad item. The probability that a good item was presented was set at .5 .

In Phase 3, the birds were given a single 1-h session in which the search key was lit prior to each presentation of a prey item. Once the search key was lit, a prey item was presented after a variable interval of $5 \mathrm{sec}$ (VI 5). A VI schedule, rather than an FI schedule, was used for the search state so that, in the experimental sessions (described below), the birds could not use the expiration of an FI with no prey items as a cue that the part of the session with choices had ended. The 12 time intervals for the VI 5 were generated according to the method of Fleschler and Hoffman (1962). A response to the search key following the VI requirement extinguished the search key and led to a presentation of either a bad or a good prey item. The VI schedule came into effect again as soon as the feeder light went out

Phase 4, consisting of two .5-h sessions, differed from Phase 3 only in that the completion of the VI 5 on the search key did not extinguish the search-key light; thus, the birds were given a choice between the search key and the prey key. The bird could accept the prey item by making one response to the prey key. The search key would then be extinguished, and the FI schedule on the prey key would come into effect. Alternatively, the bird could reject the prey item by giving three pecks to the search key. Both keys would then be extinguished for less than a second, following which the search key would come on again, signaling the beginning of a new trial.

Table 1

Procedure in Experiment 1

\begin{tabular}{|c|c|c|c|c|c|c|c|c|}
\hline & $\begin{array}{l}\text { Short } \\
\text { \& Long } \\
\text { Session }\end{array}$ & Choice & VI & $\begin{array}{c}\text { Short } \\
\text { FI }\end{array}$ & $\begin{array}{c}\text { Long } \\
\text { FI }\end{array}$ & $\begin{array}{c}p \\
\text { (Good } \\
\text { Item) }\end{array}$ & $\begin{array}{c}\text { Number of } \\
\text { Sessions }\end{array}$ & $\begin{array}{l}\text { Search } \\
\text { Key On } \\
\text { at End } \\
\end{array}$ \\
\hline \multicolumn{9}{|c|}{ Pretraining } \\
\hline \multicolumn{9}{|c|}{ (Autoshaping to respond to search key) } \\
\hline Phase 2 & No & No & No & $1 \rightarrow 2$ & $6 \rightarrow 12$ & .5 & $6-8$ & No \\
\hline Phase 3 & No & No & 5 & 2 & 12 & .5 & 1 & No \\
\hline Phase 4 & No & Yes & 5 & 2 & 12 & .5 & 2 & No \\
\hline \multicolumn{9}{|c|}{ Experimental Sessions } \\
\hline Block 1 & Yes & Yes & 5 & 2 & 12 & $.5 \rightarrow .3$ & $\begin{array}{l}12 \text { short } \\
12 \text { long }\end{array}$ & $2 \mathrm{~min}$ \\
\hline Block 2 & Yes & Yes & 5 & 2 & 12 & .3 & $\begin{array}{l}12 \text { short } \\
12 \text { long }\end{array}$ & $4 \mathrm{~min}$ \\
\hline Block 3 & Yes & Yes & 5 & 2 & 12 & .3 & $\begin{array}{l}12 \text { short } \\
12 \text { long }\end{array}$ & $4 \min$ \\
\hline Discrimination Test & \multicolumn{7}{|c|}{ (Four out of 13 long sessions cut short after $10 \mathrm{~min}$ ) } & \\
\hline
\end{tabular}


The procedure in each experimental session was the same as the one described for Phase 4 in pretraining, with one addition. At the end of each session, the search key was left on for a fixed period of time, during which the birds' keypecks were recorded but no new prey items appeared. This fixed period of time was programmed to start at the end of the first trial completed after $10 \mathrm{~min}$ in the short sessions and at the end of the first trial completed after $20 \mathrm{~min}$ in the long sessions.

Design. Each day, the birds were tested once in each box. Two of the birds received short sessions in the brown box and long sessions in the blue box; the other 2 birds received short sessions in the blue box and long sessions in the brown box. All birds were given their first session before any of the birds were given the second session. Each bird was placed in a holding cage in the experimental room between the two sessions. Every second day, the first of the two sessions was the long session, and on the other days, the first session was the short session.

The experiment consisted of three blocks of 12 days each. Data were collected following the first day in which the birds received both a long and a short session. In the first 8 days for the first block, the probability of a good item was .5. With this value, the pigeons rejected so many bad items that it might not have been possible to detect differences in acceptability of bad items between long and short sessions. To increase the frequency of accepting bad items, the probability of a good item was decreased to .3 (Lea, 1979). In the first $1 \frac{1}{2}$ blocks, the session lengths were $12 \mathrm{~min}$ for the short sessions and $22 \mathrm{~min}$ for the long sessions. Only during the first $10 \mathrm{~min}$ of short sessions and $20 \mathrm{~min}$ of long sessions did prey items follow responses to the search key. During the last $2 \mathrm{~min}$, the search key remained on but no prey items were presented. This fixed time interval at the end of the session was extended to $4 \mathrm{~min}$ in the middle of Block 2, so the session lengths were 14 and $24 \mathrm{~min}$ for short and long sessions, respectively. In the middle of Block 3, data collected over 3 days were lost because of a computer malfunction, forcing us to rerun the 3 days.

Following Block 3, the birds received a final block of sessions in which they were tested for their ability to discriminate between session lengths. This block consisted of 13 long and 13 short sessions. On the 4th, 7th, 10th, and 13th long sessions, prey items ceased to be presented after $10 \mathrm{~min}$, rather than after $20 \mathrm{~min}$, in the box in which the birds usually received long sessions. The long sessions still lasted for $24 \mathrm{~min}$, but for the last $14 \mathrm{~min}$ (rather than for the last $4 \mathrm{~min}$ ), the search key stayed on with no VI schedule in effect.

Two dependent measures were taken: (1) the proportion of bad items accepted as a function of time in the session, and (2) the number of responses to the search key in .5-min time bins at the end of $10 \mathrm{~min}$ in the short sessions, at the end of $20 \mathrm{~min}$ in the long sessions, and at the end of $10 \mathrm{~min}$ in the discrimination-test sessions.

Since the first dependent variable was binary (accept or reject), a logistic model was fitted to the proportion of bad items accepted. The analysis was conducted on an IBM PC with the use of GLIM (for details of the model-fitting procedure, see Baker \& Nelder, 1978).

\section{Results}

Within-session effects. The acceptability of bad items was greater at all times for the short sessions than for the long sessions. Figure 1 shows the mean frequency of bad items accepted over the course of the short and long sessions in the last block, before the test sessions began. The main effect of session length was significant $\left[\chi^{2}(1)=9.2\right.$, $p<.005]$. The effect of order of presentation of long and short sessions was not significant $\left[\chi^{2}(1)=0.0\right]$.

Although the programmed search time was the same in both short and long sessions (VI5), we considered the possibilities that the actual search times might differ across the two session lengths (perhaps the birds took longer to complete the VI5 in one case than they did in the other) and that the effect of session length in the last block might be attributed to a difference in the actual search time. However, the average search time was $9.70 \mathrm{sec}$ in the short sessions and $9.99 \mathrm{sec}$ in the first $10 \mathrm{~min}$ of the long sessions; this difference was not significant $[F(1,3)=$ $1.55]$.

The average frequency of accepting bad items (Figure 1) changed over time within a session. The main effect of session time, which was entered into the logistic model as a continuous variate, was significant $\left[\chi^{2}(1)\right.$ $=18.0, p<.001]$, but the interaction between session time and session length was not $\left[\chi^{2}(1)=0.4\right]$. The acceptability of bad items increased toward the end of long sessions but did not increase so much toward the end of short sessions. The acceptability of bad items was not higher at the beginning of the long sessions than it was in the middle, unlike the case in Yoerg and Kamil (1988). However, there is a suggestion of such an effect for the short sessions. A quadratic term for session length was entered into the linear model so we could detect a possible nonlinear effect of session length at the beginning of the session, but neither this effect nor its interaction with session length was significant $\left[\chi^{2}(1)=.7\right.$ and $\chi^{2}(1)=$ .5 , respectively].

Development of effect of session length. Nearly all the good items (98.8\%) were accepted throughout all blocks of the experiment. The proportions of bad items accepted for the first $10 \mathrm{~min}$ in long and short sessions over the three blocks in the experiment are shown in Figure 2. If bad items are more acceptable to the birds when they expect the session to end imminently, then a comparison of the choices in the first $10 \mathrm{~min}$ in short and long sessions should reveal an overall greater acceptability of bad items in the first $10 \mathrm{~min}$ of short sessions. In Figure 2, the increase in acceptability between Blocks 1 and 2 was due to the decrease in the frequency of good

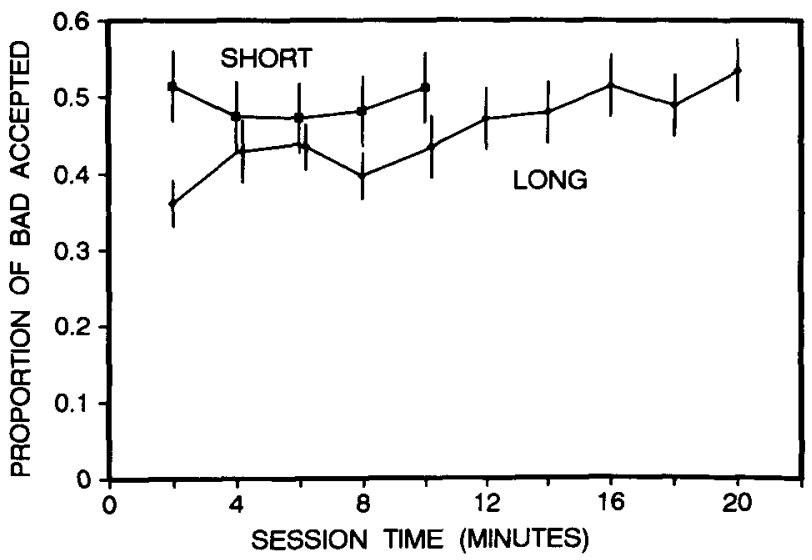

Figure 1. Average proportion of bad items that were accepted over the course of short and long sessions in the last block of days. $S E$ bars are included. 


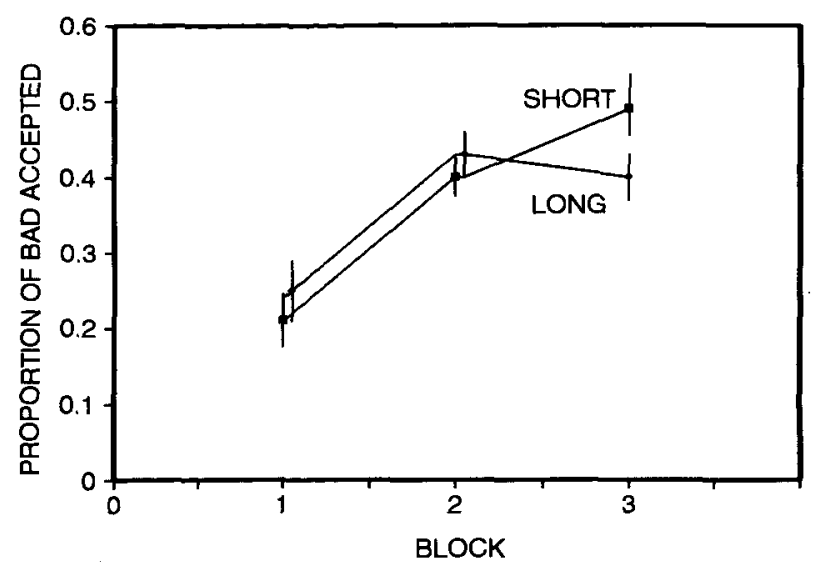

Figure 2. Average proportion of bad items accepted in first 10 min of short and long sessions for three blocks of 12 days. $S E$ bars are included.

items from .5 to .3. For 2 birds ( 3 and 11 ), no difference between long and short sessions was found prior to Block 2, and a difference in the expected direction emerged in Block 3. For another bird (26), the effect in the expected direction was evident in the last two blocks. Finally, for the last bird (14), a large difference in the opposite direction of what was expected was evident for the first two blocks but disappeared afterwards. Because of this last bird, until Block 3, the main effect of session length is not evident in the group averages presented in Figure 2. Individual differences are discussed further below. Block of days was entered into the logistic model as a continuous variate. The block $\times$ session length interaction was significant $\left[\chi^{2}(1)=12.29, p<.005\right]$.

Session-length discrimination. The number of pecks to the search key at the end of the session was taken as one measure of session-length discrimination. Weber's law predicted that the birds would respond more at the end of long sessions than at the end of short sessions, since the temporal judgment should be more accurate after the short sessions. This effect was expected to develop over time as the animals learned that one chamber was associated with short sessions and the other with long sessions. The number of pecks to the search key was averaged for the first three 30-sec time bins, because after this time, 3 birds' response rates approached the floor level. The average number of responses after $10 \mathrm{~min}$ in the short sessions and after $20 \mathrm{~min}$ in the long sessions was computed for each of the three blocks in the experiment. An analysis of variance showed neither an effect of session length $[F(1,3)=.44]$ nor an interaction with block of days $[F(1,3)=.004]$.

The discrimination-test days provide a better measure of the birds' ability to discriminate between session lengths, since responding to the search key is measured for the same time period within a session in both chambers. Figure 3 shows the mean rate of pecking after $10 \mathrm{~min}$ or the 4 temporal-discrimination-test days. The curves for the short sessions represent the average rate of responding in the short sessions on the same 4 days as the long sessions, which were cut short. As before, only the data for the first 3 half minutes were analyzed. Since the distributions were positively skewed, the data were square-root transformed. The rate of pecking after $10 \mathrm{~min}$ was higher in the chamber in which long sessions were usually given than in the chamber in which short sessions were given $[F(1,3)=10.81, p<.05]$, which showed that the birds could discriminate between the two session lengths: In the chamber in which they usually received 20-min sessions, they behaved as if they had a stronger expectation of items continuing after 10 min than they did in the chamber in which they received short sessions.

To assess the possibility that the number of responses to the search key at the end of $10 \mathrm{~min}$ was simply a function of the immediately preceding choice proportions, the correlation between the number of responses to the search key in the first .5-min time bin and the proportion of bad items accepted in the previous $2 \mathrm{~min}$ was obtained for each bird. Such a correlation would have to be negative to account for our results, since the proportion of bad items accepted in the short sessions was higher than in the long sessions, and yet the rate of responding to the search key at the end of $10 \mathrm{~min}$ in the short-session chamber was lower than the rate of responding at the end of $10 \mathrm{~min}$ in the long-session chamber. For 3 birds, the correlations were nonsignificant (Pearson $r=.26, r=-.28$, and $r=-.54$ based on $n=8$ ). For 1 bird, the correlation was significant $(r=.83, p<.01, n=8)$, but, since it was positive, it could not account for our overall pattern of results.

Individual differences. The effect of session length on choice was evident for all birds in the last block. However, for one of the birds (14), the effect was very small and only appeared after two blocks in which a large effect in the opposite direction was observed. This bird apparently did not discriminate between the two session lengths, as shown by the behavior on the temporal-discriminationtest days: No difference was found between the two cham-

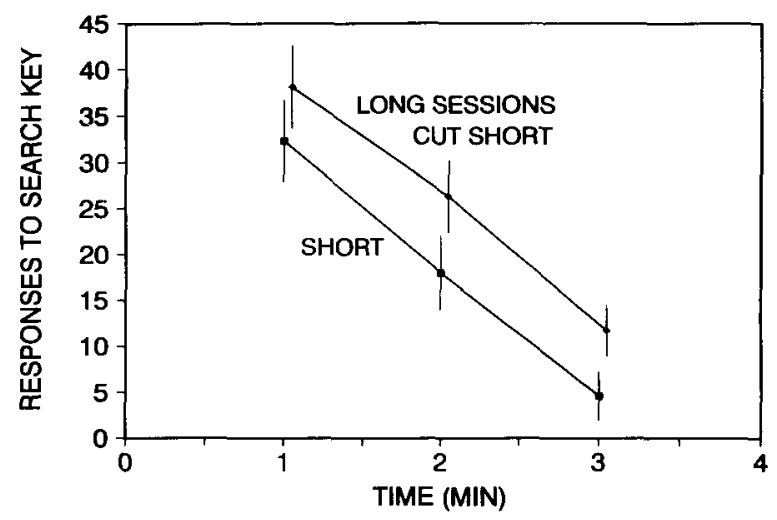

Figure 3. Average number of responses to the search key after 10 min for each bird in the chamber where long sessions usually occurred and on short sessions on the same day, for the four temporal-discrimination-test days. $S E$ bars are included. 
bers (one for short and one for long sessions) in the rate of responding to the search key at the end of $10 \mathrm{~min}$.

The 2 birds ( 3 and 11) who showed the clearest development of an effect of session length on overall proportion of bad items accepted also showed an effect of session length on the number of keypecks at the end of the session, which developed in parallel and was predicted by Weber's law.

\section{Discussion}

After approximately 24 days of sessions of each length, the birds showed a greater overall tendency to accept bad items in the short sessions than in the first $10 \mathrm{~min}$ of the long sessions.

The within-session analysis showed that the acceptability of bad items was greater at all times for short sessions. This means that during the first $10 \mathrm{~min}$ of a session, the birds could anticipate whether or not the session would extend for another $10 \mathrm{~min}$. Hence, this is an instance of "prospective timing" (i.e., responses are under the control of the time interval about to occur; Killeen \& Fetterman, 1988).

The acceptability of bad prey rose toward the end of the long sessions, as predicted by Lucas's (1987) model. This result is the opposite of what would be predicted from the results of a prey-selection study by Snyderman (1983b), who found that an increase in food deprivation in pigeons was associated with a decrease in selectivity (i.e., an increase in the ratio of bad-to-good items accepted). Assuming that, in our study, as the session progressed, the pigeons became progressively more satiated, one would predict an increase in selectivity (i.e., a decrease in the proportion of bad items accepted) rather than the opposite. Furthermore, the failure to find an order-of-session effect (first or second session in the day) on the proportion of bad items accepted suggests that satiation was not an important factor in this experiment.

In this study, we have replicated some findings on the effects of session length on prey selection that were obtained with other foraging birds (Lucas, 1987; Yoerg \& Kamil, 1988). However, in contrast to Yoerg and Kamil's (1988) study, we found no greater level in the proportion of bad items accepted at the beginning of the sessions compared with the middle of the sessions. Yoerg and Kamil note that the handling times in their study changed between bouts and suggest that the beginning of the bout effect could be interpreted as sampling. Perhaps the difference between the two studies can be attributed to our procedure of having the delays to food for good and bad items remain constant throughout the course of the experiment.

With the use of an analog of the peak procedure, we tested for discrimination between session lengths of 10 and $20 \mathrm{~min}$. Two birds responded more to the search key at the end of the long sessions than they did at the end of the short sessions, as would be predicted from Weber's law, although the main effect was not significant. Moreover, the effect for these 2 birds developed in parallel with the effect of session length on the frequency of accepting bad items.
In a more rigorous test of sensitivity to session length at the end of the experiment, all 3 birds that showed a clear effect of session length on frequency of accepting bad items also keypecked more at the end of $10 \mathrm{~min}$ in the chamber in which they usually received long sessions than they did at the end of $10 \mathrm{~min}$ in the short sessions. Although these data suggest that the birds could discriminate between session lengths, there is another interpretation. If the birds are more aroused in the box in which long sessions are administered, perhaps by virtue of the greater total amount of reward in the long sessions, then the pattern of responding at the end of $10 \mathrm{~min}$ in the two boxes (Figure 3 ) would be expected. Hence, the birds may perhaps discriminate between factors that are correlated with the differences in session length in our experiment rather than discriminate between differences in session length per se.

\section{EXPERIMENT 2}

Experiment 2 addressed the question of whether pigeons are sensitive to variability in session length. A comparison of choice behavior in sessions of fixed and variable session length, with the mean length the same in both cases, was expected to reveal differences that depended on time within a session (J. R. Lucas, personal communication, January 5, 1990). At the beginning of the sessions, it was expected that a higher proportion of bad items would be accepted in the variable sessions, because the probability of the bout ending in the near future is higher in the variable sessions than in the fixed ones. However, toward the end of the sessions of fixed length, the reverse pattern ought to occur: A lower proportion of bad items should be accepted in the variable sessions, because the probability of the bout extending beyond the mean is higher in the variable sessions than in the fixed ones (Lucas, 1987).

As in the previous experiment, birds were given two sessions on each day. One of the sessions, in a distinctively marked chamber, was of fixed length: Session length was always $20 \mathrm{~min}$. The other session, in another distinctively marked chamber, was of variable length: Session length could vary from as little as $5 \mathrm{~min}$ to as long as $35 \mathrm{~min}$, but the average length was $20 \mathrm{~min}$.

The methods were essentially the same as in the previous experiment. An attempt was made to make the different boxes more discriminable by having different colored houselights illuminate each box instead of having colored paper on the response panels. Also, the two sessions were temporally separated to a greater extent: In Experiment 1, the second session usually followed after $1.5 \mathrm{~h}$, but in Experiment 2 , it usually followed after approximately $4 \mathrm{~h}$.

\section{Method}

Subjects. Subjects were 6 new White King pigeons with various preexperimental histories. All had keypecked before.

Pretraining and Procedure. The pretraining and procedure were the same as in Experiment 1, with minor modifications. In Phase 1 of pretraining, the birds were autoshaped to peck the two colors of the prey keys, as well as the search key. Phase 4 of pretraining 
was identical to a regular experimental session in that the search key was left on for $4 \mathrm{~min}$ at the end of the session, but responses to the search key did not lead to the presentation of new prey items. The time period at the end of each session remained 4 min long for the duration of the experiment. Following pretraining sessions, the experiment began, and each bird received two sessions each day: one in the fixed-session-length box and one in the variablesession-length box. The probability of a good item was .3 for the duration of the experiment.

For 3 of the birds, the good prey color was green and the bad prey color was red; for the other 3 birds, the good prey color was red and the bad was green.

Design. On each day, the birds were tested once in each box. Three of the birds received fixed sessions in a box with a blue houselight and variable sessions in a box with a yellow houselight; the other 3 birds received fixed sessions in the yellow-lit box and long sessions in the blue-lit box. Every second day, the first of the two sessions was the variable session; on the other days the first session was the fixed session.

The seven session lengths in the variable box were: $5,10,15$, $20,25,30$, and $35 \mathrm{~min}$. The experiment consisted of four blocks of 14 days each. In each block, each of the seven session lengths in the variable box was presented once as the first session in the day and once as the second session. One random order of the seven session lengths in the variable box was generated and repeated twice for each block of days for 4 of the birds. The other 2 birds received an order in which short sessions occurred when the other 4 birds received long sessions, and vice versa. The order of session lengths in the variable box within a block was the same for each block.

Immediately following the fourth block, the birds received a final block of sessions that included four (rather than only two) 20min sessions in each box. In between each of these 20 -min sessions were only one very short $(5 \mathrm{~min})$ and one very long $(35 \mathrm{~min})$ sessions.

\section{Results}

Within-session effects. The birds accepted a higher proportion of bad items in the variable box than in the fixed box. This effect did not reverse toward the end of the session, as expected, but it did diminish. Figure 4 shows the proportion of bad items accepted over the course of 20-min sessions in the fixed and variable boxes in Block 4 (Figure 4a) and in the last block, in which there were four 20 -min sessions in each box rather than just two (Figure 4b). For Block 4, the interaction of time within the session (first vs. second half of the session) and fixed-versus variable-session length was significant $\left[\chi^{2}(1)=8.1, p<.005\right]$. For the last block, the interaction was in the expected direction but failed to reach significance $\left[\chi^{2}(1)=3.15\right]$. For Block 4 , there was a significant effect of the order of presentation of the sessions. It was the opposite of a satiation effect-a higher proportion of bad items was accepted in the second session of the day, compared with the first $\left[\chi^{2}(1)=41.23\right.$, $p<.005]$. The overall choice proportions for the first sessions of the day were .35 for fixed sessions and .45 for variable sessions; the choice proportions for the second sessions of the day were .48 for fixed sessions and .59 for variable sessions. The effect did not interact with session type $\left[\chi^{2}(1)=2.1\right]$. For the last block, the effect of the order of presentation of fixed and variable sessions was not significant $\left[x^{2}(1)=0.4\right]$.
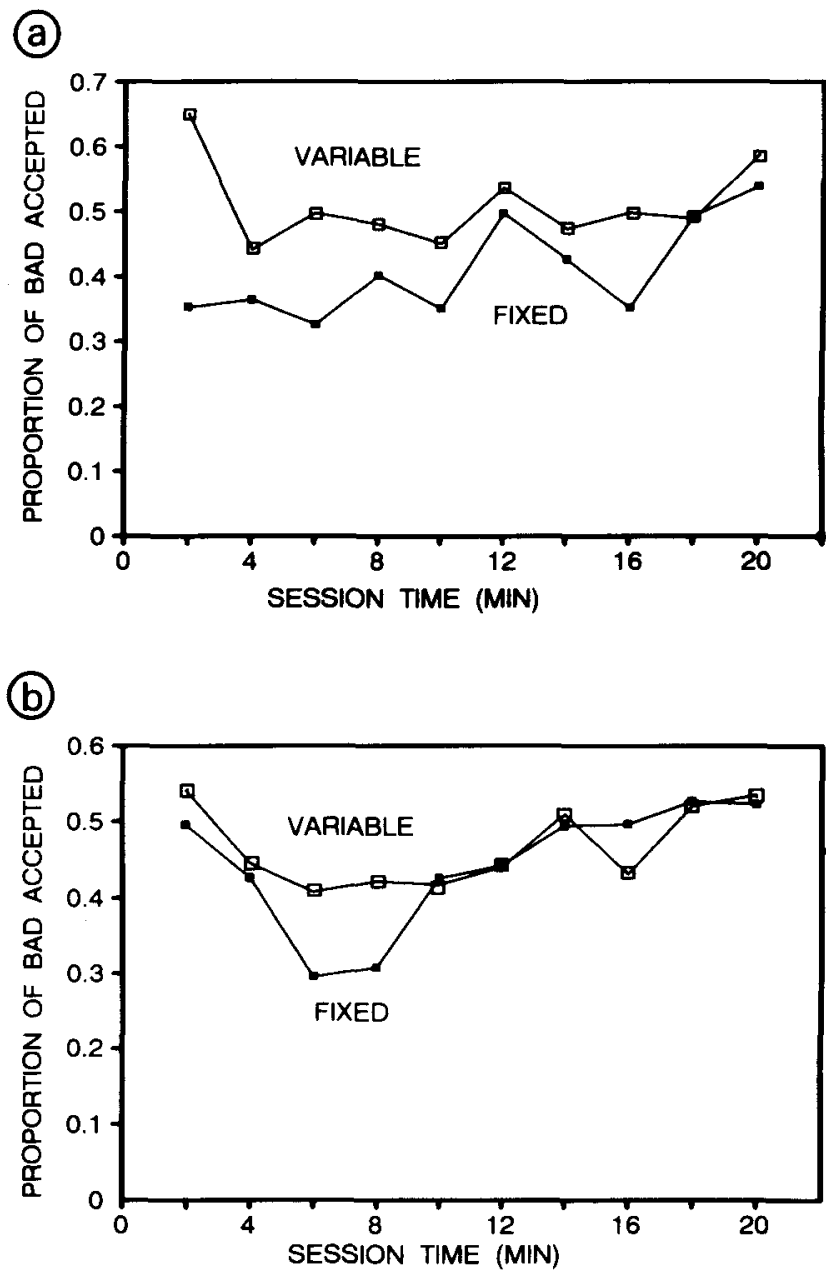

Figure 4. Proportion of bad items accepted over the course of 20 min sessions in the fixed- and variable-session boxes (a) in the two sessions in Block 4 (b) in the four 20-min sessions in the last block. For both the first and second halves of fixed and variable sessions, the standard error was .05 in (a) and .06 in (b).

The average actual search time (as opposed to programmed search time, which was VI5) in the 20-min sessions in Block 4 was $7.6 \mathrm{sec}$ in the fixed sessions and $6.8 \mathrm{sec}$ in the variable sessions. This difference was not significant $[F(1,5)=1.05]$.

Development of effect of variability in session length. As in Experiment 1, nearly all the good items (97.7\%) were accepted throughout all blocks of the experiment. Figure 5a shows the overall proportion of bad items accepted in 20-min sessions for each of the four blocks of days in the fixed- and variable-session-length boxes. A higher proportion of bad items accepted is evident in the variable box, and this effect developed over the four blocks of days [interaction of block $\times$ session type; $\chi^{2}(1)$ $=7.8, p<.01]$. The same effect is evident if only the first 5 min of all sessions are considered. Figure $5 b$ shows the overall proportion of bad items accepted in the first 
(a)

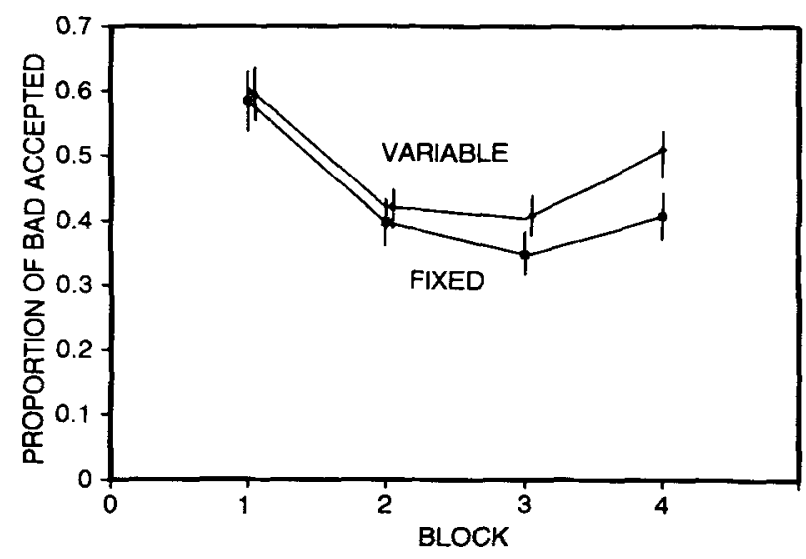

(b)

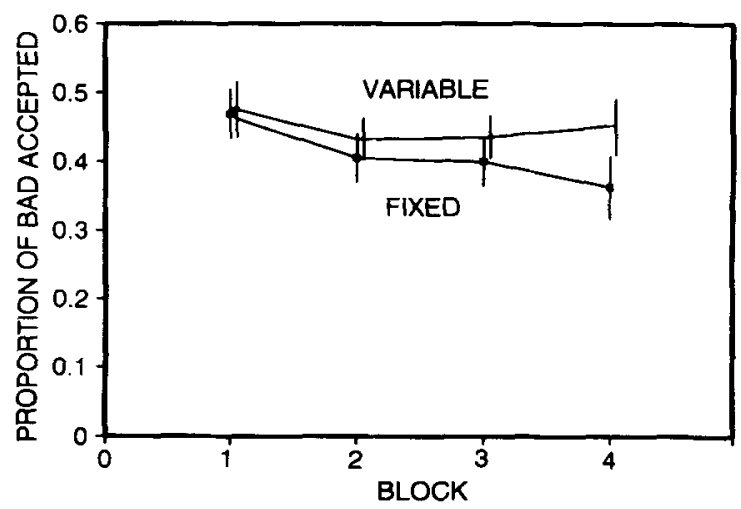

Figure 5. Average proportion of bad items accepted in the fixedand variable-session-length boxes for each of 4 blocks of days in (a) 20-min sessions (b) the first 5 min of all sessions. $S E$ bars are included.

5 min of all sessions for each of the four blocks of days in the fixed- and variable-session-length boxes. A higher proportion of bad items accepted is evident in the variable box, and this effect develops over the four blocks of days [interaction of block $\times$ session type; $\chi^{2}(1)=7.9$, $p<.005]$.

Temporal discrimination in variable sessions. In the box with variable sessions, the probability of the session ending increases with session length. For example, the conditional probability of the session extending beyond $10 \mathrm{~min}$, given that it has already lasted $10 \mathrm{~min}$, is .83 $(5 / 6)$. If the session has already lasted $25 \mathrm{~min}$, then this conditional probability is reduced to $.67(2 / 3)$. If the birds are sensitive to this property of session length in the variable box, they should respond more to the search key at the end of the choice period the earlier it is. The number of responses to the search key in the first $1.5 \mathrm{~min}$ at the end of the sessions of various lengths in the variablesession box are shown for Block 4 (Figure 6). The rate of responding to the search key decreased with increasing session length $[F(6,30)=5.00, p<.005]$. Neither an effect of order of session $[F(1,5)=1.84]$ nor an interaction of session length with order $[F(6,30)=1.59]$ was found. The increase in rate of responding from 30min sessions to 35-min sessions was evident for 2 birds only and cannot be accounted for.

In the last block of days, the rate of responding to the search key in the first $1.5 \mathrm{~min}$ at the end of 20 -min sessions did not differ in the fixed-and the variable-session boxes. An analysis of variance on the square-root transformed data showed neither a main effect of fixed- versus variable-session length $[F(1,3)=3.25]$ nor an interaction with block of days $[F(3,15)=.210]$.

Individual differences. In Block 4, in the first half of the 20-min sessions, all birds accepted more bad items, on the average, in the variable sessions than in the fixed ones. The differences in the proportions ranged from .025 to .255 . Five of the 6 birds accepted more bad items overall in the variable sessions than in the fixed ones. However, the development of this effect over the four blocks of days, as shown for the group average in Figure 5, was only clear for 2 of the birds.

For the measure of temporal discrimination in the variable sessions in Block 4, all birds showed a general decrease in responding to the search key with session length.

\section{Discussion}

A comparison of the choice proportions in the first $5 \mathrm{~min}$ of sessions in the fixed- and the variable-session boxes showed that the birds exhibited a sensitivity to variability in session length after approximately $\mathbf{4 2}$ days of experience in each box. The birds were apparently also sensitive to elapsing time within the session, as shown by their rate of responding to the search key (Figure 6) at the end of sessions of different lengths.

The within-session analysis showed that the first part of the prediction of optimal prey selection was verified:

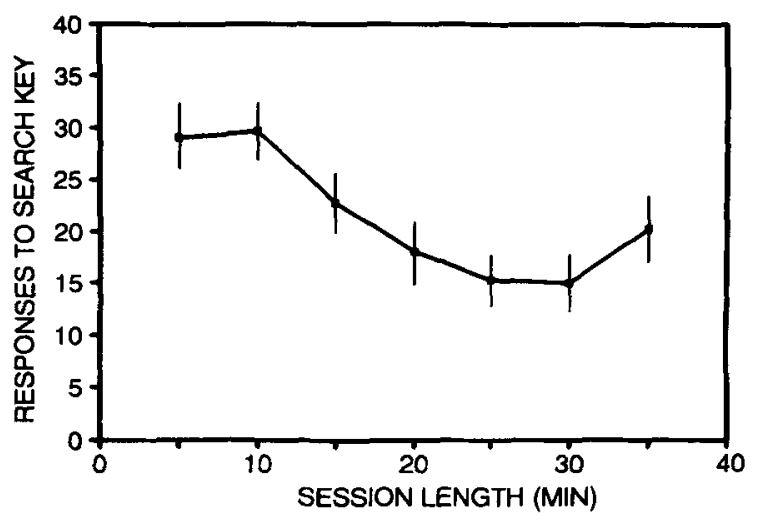

Figure 6. Average number of responses to the search key in $1.5 \mathrm{~min}$ at the end of sessions of various lengths (ranging from 5 to $35 \mathrm{~min}$ ) in the variable-session box in Block 4 . $S E$ bars are included. 
At the beginning of the 20-min sessions, the birds accepted a higher proportion of bad items in the variable-session box than in the fixed-session box. However, the second part of the prediction, that this effect would reverse toward the end of the session, was not verified, although the difference in choice proportions between the two boxes did diminish.

\section{GENERAL DISCUSSION}

In both experiments, pigeons showed that they could discriminate among session lengths and that they could adjust their foraging behavior, depending on time horizon, in accordance with the predictions of optimal prey selection. However, one prediction of optimal prey selection was not verified. In Experiment 2, the birds did not accept a higher proportion of bad items toward the end of $20 \mathrm{~min}$ in the fixed-session chamber than they did in the variable-session chamber. Why the prediction failed is a question that remains to be answered.

Session length has not been considered as an important aspect of experimental design in operant experiments. In many studies, the end of a session is defined as a fixed number of reinforcers rather than as a fixed time. The finding that choice behavior can depend on session length and on variability in session length suggests that in studies of choice behavior, session length might be better defined as a fixed time. In another example of the effect of session length on operant behavior, Elliffe and Davison (1985) have reported that response rates on multiple schedules in long sessions are lower than those in short sessions.

One question that remains to be investigated is in what kinds of situations sensitivity to session length affects behavior. Session length had no effect on choice behavior in two separate studies on a different foraging situation: the two-armed bandit (two concurrent random-ratio schedules). In one study, the session lengths were 50 versus 250 choices (Shettleworth \& Plowright, 1989); in the other, they were 3 versus $12 \mathrm{~min}$ (Reid, 1988). Given that, in this study, pigeons can discriminate between 10and 20-min sessions, the question arises as to whether discrimination of these two session lengths is task-specific (i.e., does not apply to the two-armed bandit) or whether, in the two-armed bandit, the birds can discriminate between session lengths, yet do not adjust their foraging behavior accordingly.

The results of these experiments also have implications with respect to timing mechanisms. It has been suggested that pigeons use a "resetting timing strategy" (Roberts, Cheng, \& Cohen, 1989), whereby reinforcements serve as a cue for clock resetting. Our results suggest that pigeons are capable of using two separate timing mechanisms: one for time intervals within an experimental session and one for session length itself. The timing mechanism for session length is currently under investigation.

\section{REFERENCES}

ABARCA, N., \& FANTINo, E. (1982). Choice and foraging. Journal of the Experimental Analysis of Behavior, 38, 117-123.

BAKer, R. J., \& Nelder, R. A. (1978). The GLIM system, Release 3: Generalised linear interactive modeling. Oxford, U.K.: Numerical Algorithms Group.

BARNARD, C. M., HURst, J. L. (1987). Time constraints and prey selection in common shrews (Sorex araneus L.). Animal Behaviour, 35, $1827-1837$

CATANIA, A. C. (1970). Reinforcement schedules and psychophysical judgments: A study of some temporal properties of behavior. In W. N. Schoenfield (Ed.), The theory of reinforcement schedules (pp.1-42). New York: Appleton-Century-Crofts.

СнURCh, R. M. (1978). The internal clock. In S. Hulse, H. Fowler, \& W. K. Honig (Eds.), Cognitive processes in animal behavior (pp. 277-310). Hillsdale, NJ: Erlbaum.

Dow, S. M., \& LEA, S. E. G. (1987). Sampling of schedule parameters by pigeons: Test of optimizing theory. Animal Behaviour, 35, 102-114.

Dreyfus, L. R., Fetterman, J. G., Smith, L. D., Stubes, D. A. (1988). Discrimination of temporal relations by pigeons. Journal of Experimental Psychology: Animal Behavior Processes, 14, 349-367.

Eluffe, D., \& DAvison, M. (1985). Performance in continuously available multiple schedules. Journal of the Experimental Analysis of Behavior, 44, 343-353.

Fantino, E., Abarca, N. (1985). Choice, optimal foraging, and the delay-reduction hypothesis. Behavioral \& Brain Sciences, 8, 315-330.

Fleschler, M., HoffMaN, H. (1962). A progression for generating variable-interval schedules. Journal of the Experimental Analysis of Behavior, 5, 529-530.

Gibbon, J., Allan, L. (Eds.) (1984). Timing and time perception. Annals of the New York Academy of Science, Vol. 423

Hanson, J., \& Green, L. (1989). Foraging decisions: Prey choice by pigeons. Animal Behaviour, 37, 429-443.

houston, A. I., Kacelnik, A., McNamara, J. M. (1982). Some learning rules for acquiring information. In D. McFarland (Ed.), Functional ontogeny (pp. 140-191). Boston: Pitman Advanced Publishing Program.

Killeen, P. R., Fetterman, J. G. (1988). A behavioral theory of timing. Psychological Review, 95, 274-295.

Krebs, J. R., Erichsen, J. T., Webber, M. I., Charnov, E. L. (1977). Optimal prey selection in the great tit (Parus major). Animal Behaviour, 25, 30-38.

LEA, S. E. G. (1979). Foraging and reinforcement schedules in the pigeon: Optimal and non-optimal aspects of choice. Animal Behaviour, 27, 875-886.

LUCAS, J. R. (1985). Time constraints on diet choice: Different predictions from different constraints. American Naturalist, 126, 680-705.

LuCAS, J. R. (1987). The influence of time constraints on diet choice of the great tit, Parus major. Animal Behaviour, 35, 1538-1548.

LucAs, J. R. (1990). Time scale and diet choice decisions. In R. N. Hughes (Ed.), Behavioural mechanisms of food selection (NATO ASI Series, Series A: Life Sciences). Berlin: Springer-Verlag.

McNamara, J. M., Houston, A. I. (1987). Partial preferences and foraging. Animal Behaviour, 35, 1084-1099.

REID, P. (1988). The effect of time constraints on performance in bandit problems. Unpublished manuscript.

ROBERTS, S. (1981). Isolation of an internal clock. Joumal of Experimental Psychology: Animal Behavior Processes, 7, 242-268.

Roberts, W. A., Cheng, K., \& Cohen, J. S. (1989). Timing light and tone signals in pigeons. Joumal of Experimental Psychology: Animal Behavior Processes, 15, 23-35.

SHETTLEWORTH, S. J. (1988). Foraging as operant behavior and operant behavior as foraging: What have we learned? In G. Bower (Ed.), The psychology of learning and motivation: Advances in research and theory (Vol. 22). London: Academic Press.

Shettleworth, S. J., \& Plowright, C. M. S. (1989). Time horizons of pigeons on a two-armed bandit. Animal Behaviour, 37, 610-623. 
Snyderman, M. (1983a). Optimal prey selection: Partial selection, delay of reinforcement and self control. Behaviour Analysis Letters, 3, 131-147.

SNYDERMAN, M. (1983b). Optimal prey selection: The effects of food deprivation. Behaviour Analysis Letters, 3, 359-369.

StePhens, D. W., \& KREBS, J. R. (1986). Foraging theory. Princeton, NJ: Princeton University Press.

Swennen, C., Leopold, M. F., de Bruijn, L. L. M. (1989). Timestressed oystercatchers, Haematopus ostralegus, can increase their intake rate. Animal Behaviour, 38, 8-22.
Timberlake, W., Gawley, D. J., \& Lucas, G. A. (1987). Time horizons in rats foraging for food in temporally separated patches. Journal of Experimental Psychology: Animal Behavior Processes, 13, 302-309.

Yoerg, S. I., \& KAMIL, A. (1988). Diet choices of blue jays (Cyanocitta cristata) as a function of time spent foraging. Journal of Comparative Psychology, 102, 230-235.

(Manuscript received July 1, 1990;

revision accepted for publication January 25, 1991.) 